\title{
Lessons Learned from Low-Frequency Noise Studies on Fully Depleted UTBOX Silicon-on-Insulator nMOSFETs
}

E. Simoen ${ }^{\mathrm{a}}$, M. Aoulaiche ${ }^{\mathrm{a}}$, S. D. dos Santos ${ }^{\mathrm{b}}$, J.A. Martino ${ }^{\mathrm{b}}$, V. Strobel ${ }^{\mathrm{c}}$, B. Cretu ${ }^{\mathrm{c}}$, J.-M. Routoure ${ }^{c}$, R. Carin ${ }^{c}$, A. Rodríguez Luque ${ }^{\mathrm{d}}$, J.A. Jiménez Tajada ${ }^{\mathrm{d}}$ and C. Claeys ${ }^{\mathrm{a}, \mathrm{e}}$

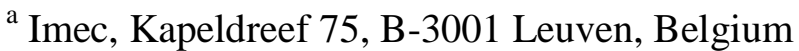

${ }^{\mathrm{b}}$ LSI/PSI/USP - University of São Paulo, Brazil

${ }^{\mathrm{c}}$ GREYC/ENSICAEN/CNRS UMR 6072/ Université de Caen - Basse Normandie, France

${ }^{\mathrm{d}}$ Universidad de Granada : Departamento de Electrónica y Tecnología de los Computadores, Facultad de Ciencias, Granada, Spain

e also at EE Depart. KU Leuven, Kasteelpark Arenberg 10, B-3001 Leuven, Belgium

The low-frequency (LF) noise behavior of Fully Depleted (FD) Ultrathin Buried Oxide (UTBOX) Silicon-on-Insulator (SOI) nMOSFETs is described from the perspective of the three major noise sources: 1/f-like or flicker noise, associated with carrier trapping/detrapping in the gate oxide; Generation-Recombination (GR) noise due to processing-induced defects in the thin silicon film and single-oxide-trap-related Random Telegraph Noise (RTN). The fully depleted nature of the thin silicon films $(<20 \mathrm{~nm})$ offers the unique opportunity to study and demonstrate the front-back coupling of the $1 / \mathrm{f}$ noise. At the same time, a large variability is induced in the noise magnitude by the Lorentzian noise, associated with GR events through defects in the silicon film. A method to discriminate oxide- from film-defects related Lorentzian noise is pointed out. Finally, the implications for future fully depleted fintype of devices will also be discussed.

\section{Introduction}

As device dimensions in Complementary Metal-Oxide-Semiconductor (CMOS) technology scale further down along the roadmap, variability of the main static parameters, like the threshold voltage $\mathrm{V}_{\mathrm{T}}$ becomes a big issue, threatening the proper functioning of logic and memory circuits (1-3). One of the main contributors to the variability in planar bulk technologies are the Random Dopant Fluctuations (RDF), which find their root cause in the random nature of ion implantation, used for engineering the channel doping concentration and, hence, the $\mathrm{V}_{\mathrm{T}}$. This can be largely avoided by going over to a fully depleted (FD) Silicon-on-Insulator (SOI) or a multiple-gate fin-type of platform, where a natural-doped silicon layer is used instead (4,5). Non-intentionally doped FD Ultra-thin Buried Oxide (UTBOX) SOI offers several advantages from a viewpoint of the suppression of dopant-related variability and short-channel effects (5). There is also interest in using such devices as capacitorless 1-transistor (1T) floatingbody RAM (1T-FBRAM) cells $(6,7)$. For the latter applications, charge retention is one of the critical parameters, requiring a tight control of defect-related carrier generation and recombination (GR). One of the techniques which lends itself nicely to this purpose is low-frequency (LF) noise spectroscopy, which can reveal GR centers in the gate 
dielectric or the silicon film $(8,9)$. Three main types of low-frequency noise can be distinguished, namely, 1/f-like or flicker noise, GR noise and Random Telegraph Noise (RTN). Each of these three noise sources will be discussed here for UTBOX SOI nMOSFETs,

Another well-known source of dynamic fluctuations appears at the horizon, which is related to the occurrence of Random Telegraph Noise (RTN) in small-area transistors (10-13). In the past, RTN was only relevant for analog and mixed signal applications but currently it can become problematic even for deeply scaled logic and memory applications as well $(3,14,15)$. The origin of RTN is related to charge capture and emission by a single trap, residing generally in the gate dielectric of a MOS device (10). Ample evidence has been presented that the corresponding normalized amplitude of the drain current fluctuation $\left(\Delta \mathrm{I}_{\mathrm{D}} / \mathrm{I}_{\mathrm{D}}\right)$ can range over several orders of magnitude (16-21). While this wide variation was puzzling at first, it has become clear that it can be understood in the frame of a non-uniform filamentary channel, defined by the random location of dopant atoms and fixed oxide charges, with a trap in its neighborhood (1$3,18,19)$. In this picture, the spread in RTN amplitude is mainly defined by the trap position with respect to the non-uniform potential landscape of the channel.

Here, it will be demonstrated that for thin-film, fully depleted transistors, e.g., FD SOI or narrow fin-type of devices (bulk or SOI FinFETs) there is an alternative source of variability of RTN - or in general Lorentzian Generation-Recombination (GR) noise which is associated with the energy level position of a GR center in the silicon film. It is shown that this is related to the fact that in FD structures, the quasi Fermi level for electrons $\mathrm{E}_{\mathrm{Fn}}$ can be easily swept over a large portion of the band gap by the front and/or back-gate voltage, so that its relative position with respect to the trap level $\mathrm{E}_{\mathrm{T}}$ can change significantly. This gives rise to a Lorentzian noise amplitude which may vary over more than a decade. This sensitivity to voltage variations helps to understand the device-todevice variation in the current noise power spectral density (PSD) at the same biasing condition. This will be illustrated here for nMOSFETs made in UTBOX SOI wafers. If at the same time, a thin silicon film is used, coupling effects between the front and the back or buried interface start to play (22). As is shown here, for a $t_{\mathrm{Si}}<20 \mathrm{~nm}$, coupling in the LF noise (and in particular in the $1 / \mathrm{f}$ noise) becomes observable $(23,24)$. A procedure to derive the correct front-channel noise PSD will be proposed.

\section{Experimental details}

The FD SOI nMOSFETs studied have been fabricated on SOI substrates with nominally $10 \mathrm{~nm}$ BOX and 20 or $10 \mathrm{~nm}$ Si film thickness $\left(\mathrm{t}_{\mathrm{Si}}\right)$. Transmission Electron Microscopy (TEM) reveals that the actual values for the short-channel devices studied are closer to 18 $\mathrm{nm}(\mathrm{BOX})$ and 14 and $6 \mathrm{~nm}$ for $\mathrm{t}_{\mathrm{si}}$. A cross-section micrograph is depicted in Fig. 1, indicating the main device dimensions. Different types of gate stack have been investigated with $5 \mathrm{~nm}$ thermal $\mathrm{SiO}_{2}\left(\mathrm{t}_{\mathrm{ox}}\right)$ as the reference, but also $\mathrm{HfO}_{2}$-based high-k devices have been analyzed. Processing splits with or without extensions have been compared, whereby extensionless structures may offer a higher retention time (6). The device width $\mathrm{W}=1 \mu \mathrm{m}$ and different effective lengths $(105 \mathrm{~nm}$ or $69 \mathrm{~nm})$ were studied. Noise measurements have been mainly performed in linear operation $\left(\mathrm{V}_{\mathrm{DS}}=0.05 \mathrm{~V}\right)$ and with the back-gate grounded. The front gate voltage $\left(\mathrm{V}_{\mathrm{GS}}\right)$ was stepped from weak to 
strong inversion in 50 or $100 \mathrm{mV}$ steps. Also noise in the back channel was measured with the front gate at $0 \mathrm{~V}$. In a few cases, corresponding with $\mathrm{t}_{\mathrm{Si}}=14 \mathrm{~nm}$, the noise in the front (back) channel was evaluated with the opposite interface biased in accumulation.

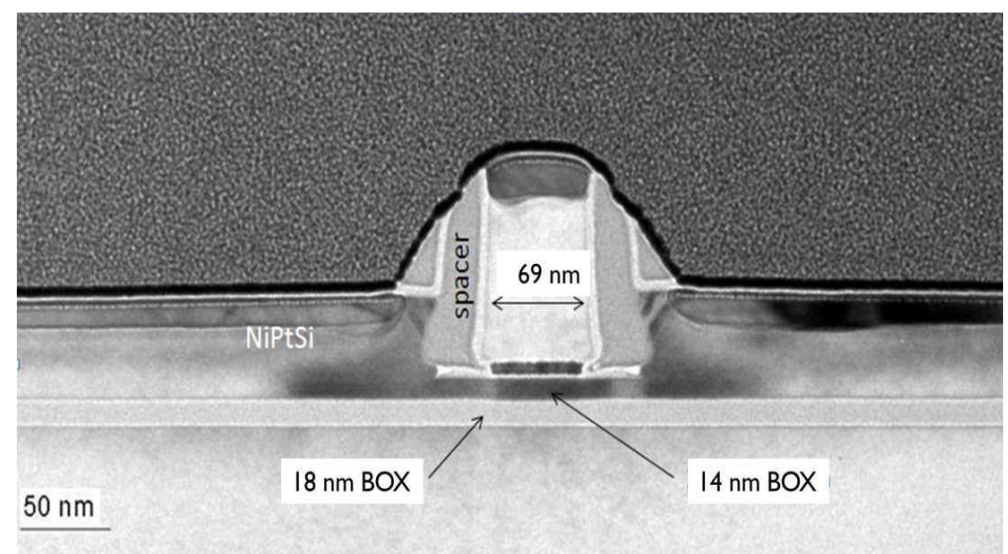

Figure 1. Transmission Electron Microscopy cross-section of a $69 \mathrm{~nm}$ long UTBOX nMOSFET with a $14 \mathrm{~nm}$ Si film and $18 \mathrm{~nm}$ BOX thickness.

\section{Flicker noise and coupling}

Typical front-channel noise spectra of the current noise PSD $S_{I}$ versus frequency $f$ exhibit both 1/f-like noise and Lorentzian noise, like in Fig. 2. The observed 1/f-like noise is dominated by number fluctuations, i.e., it originates from trapping in the gate oxide, both for the front-gate and back-gate noise PSD (24). This is illustrated by Fig. 3, representing the normalized current PSD $\left(S_{I} / I_{D}{ }^{2}\right)$ versus $I_{D}$ in linear operation and comparing it with $\left(\mathrm{g}_{\mathrm{m}} / \mathrm{I}_{\mathrm{D}}\right)^{2}$. As can be seen, both functions are proportional to it other, with a plateau in weak inversion. This is a fingerprint of the $\Delta \mathrm{n}$ or number fluctuations origin of the $1 / \mathrm{f}$ noise $(25,26)$. The presence of the Lorentzian noise at higher frequencies in Fig. 2 may also indicate noise due to traps in the gate oxide.

The fact that the flicker noise is due to trapping implies that a density of oxide traps $\mathrm{N}_{\mathrm{ot}}$ can be extracted from the input-referred voltage noise PSD $\left(\mathrm{S}_{\mathrm{VG}}=\mathrm{S}_{\mathrm{I}} / \mathrm{g}_{\mathrm{m}}{ }^{2}\right)$ at flat band voltage $\left(\mathrm{V}_{\mathrm{FB}}\right)$, according to $(25,26)$ :

$$
S_{V F B_{1,2}}=\frac{q^{2} k T \lambda N_{o t_{1,2}}}{f^{Y} W L C_{o x_{1,2}}^{2}}
$$

with $q$ the elementary charge, WL the device effective width times effective length, $\gamma$ the frequency exponent $(\sim 1)$, $\mathrm{k}$ Boltzmann's constant, $\mathrm{T}$ the absolute temperature and $\lambda$ the electron tunneling depth in the gate dielectric $\left(\sim 0.1 \mathrm{~nm}\right.$ for $\left.\mathrm{SiO}_{2}\right) . \mathrm{C}_{\mathrm{ox}}$ is the oxide capacitance density of the front (subscript 1) and the back (subscript 2) interface. However, in the case of thin-film fully depleted SOI, the front-channel noise PSD will be affected by coupling to the noise at the back-channel $(22,27)$, giving rise to: 


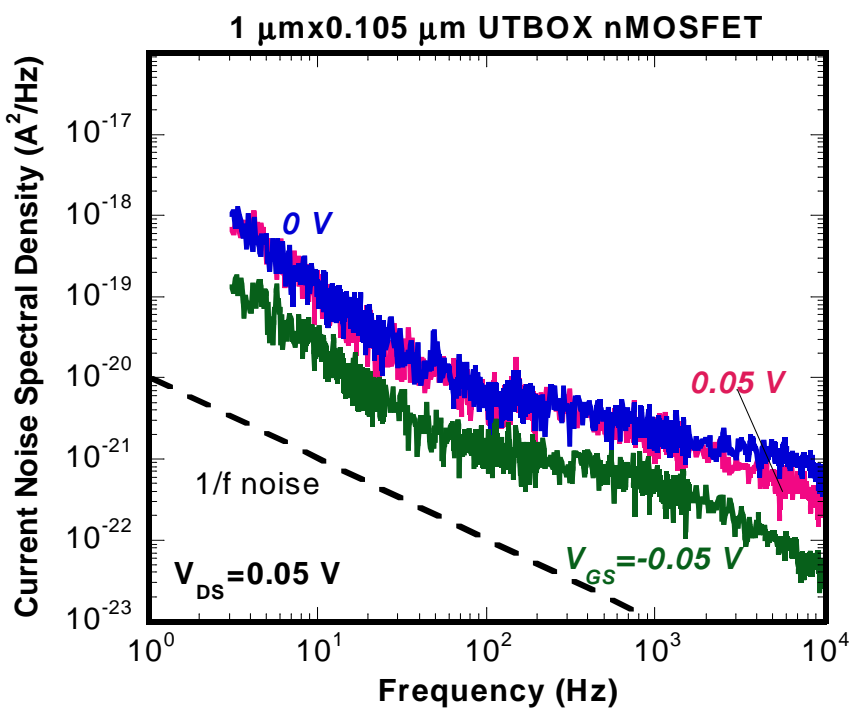

Fig. 2. Low-frequency noise spectra around $\mathrm{V}_{\mathrm{T}}$ for a $1 \mu \mathrm{mx} 0.105 \mu \mathrm{m}$ UTBOX SOI nMOSFET, exhibiting flicker noise around $10 \mathrm{~Hz}$. The back-gate is at $0 \mathrm{~V}$ in depletion. The drain voltage $\mathrm{V}_{\mathrm{DS}}=0.05 \mathrm{~V}$ in linear operation.

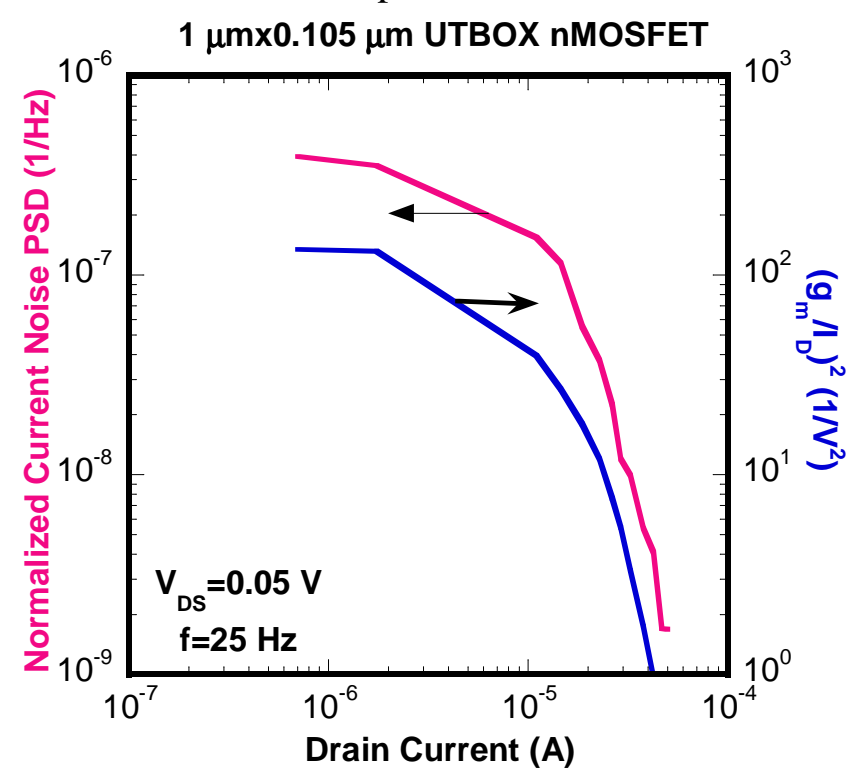

Fig. 3. Normalized current noise PSD at $25 \mathrm{~Hz}$ versus drain current and $\left(\mathrm{g}_{\mathrm{m}} / \mathrm{I}_{\mathrm{D}}\right)^{2}$ versus $\mathrm{I}_{\mathrm{D}}$ in linear operation $\left(\mathrm{V}_{\mathrm{DS}}=0.05 \mathrm{~V}\right)$ for a $1 \mu \mathrm{mx} 0.105 \mu \mathrm{m}$ UTBOX SOI nMOSFET.

$$
S_{I}^{n \infty p} \approx S_{I}^{\alpha \omega}\left(1+\alpha^{2} \frac{C_{\sigma x, 1}^{2} N_{o t, 2}}{C_{\sigma x, 2}^{2} N_{o t, 1}}\right)
$$

with $\alpha$ corresponding with:

$$
\alpha=\frac{C_{S i} C_{o x, 2}}{C_{o x, 1}\left(C_{o x, 2}+C_{S i}+C_{i t_{1} 2}\right)}
$$


In Eq. [3], $\mathrm{C}_{\mathrm{Si}}$ is the capacitance density of the silicon film $\left(=\varepsilon_{0} \varepsilon_{\mathrm{Si}} / \mathrm{t}_{\mathrm{si}} ; \varepsilon_{0}\right.$ the permittivity of vacuum and $\varepsilon_{\mathrm{Si}}$ the dielectric constant of silicon). In case the back-interface state density $\mathrm{C}_{\mathrm{it}, 2}$ can be neglected, Eq. [3] can be simplified to:

$$
\alpha=\frac{C_{S i}}{C_{o x, 1}\left(1+\frac{C_{S i}}{C_{o x, 2}}\right)}
$$

In Eq. [2], $S_{I}{ }^{\text {dep }}$ and $S_{I}^{\text {acc }}$ are the current noise PSD with the back interface in depletion or in accumulation. In case the back (or front) interface can be biased in accumulation, the fluctuations by back (front) oxide traps can be screened completely, so that only the noise due to traps at the front (or back) interface will be measured, enabling a correct extraction of $\mathrm{N}_{\mathrm{ot}}$, using Eq. [1]. In case of ultra-thin films, where the back interface cannot be accumulated, Eq. [2] predicts that the measured noise at the front will be higher due to the effect of the back oxide traps. In case the trap density is the same, the enhancement factor will be $\alpha^{2} \mathrm{t}^{2}{ }_{\text {ox }, 2} / \mathrm{t}^{2}$ ox,1 .

Figure 4 represents the $S_{\mathrm{VG}}$ at flat-band for the back channel versus the frontchannel 1/f noise PSD for a number of $69 \mathrm{~nm}$ UTBOX nMOSFETs with $\mathrm{t}_{\mathrm{si}} \sim 14 \mathrm{~nm}$ and $\mathrm{t}_{\mathrm{ox}}=5 \mathrm{~nm}$. In both cases, the channel noise was measured with the opposite interface at 0 $\mathrm{V}$, thus in depletion. The dashed line represents a linear fit corresponding with a slope of 8.5 (24), which is smaller than the anticipated ratio of $\sim 13$ for equal trap densities $\mathrm{N}_{\mathrm{ot}, 1}=\mathrm{N}_{\mathrm{ot}, 2}$. This points to the impact of the front-back coupling, on the one hand and a difference in oxide trap density, on the other. In this case, a correct evaluation of $\mathrm{N}_{\mathrm{ot}}$ is possible by measuring the noise with the opposite interface in accumulation. Typical trap densities are in the range $2 \times 10^{16} \mathrm{~cm}^{-3} \mathrm{eV}^{-1}$ to $2 \times 10^{17} \mathrm{~cm}^{-3} \mathrm{eV}^{-1}$ for the front and $2 \times 10^{16} \mathrm{~cm}^{-}$ ${ }^{3} \mathrm{eV}^{-1}$ to $1.3 \times 10^{17} \mathrm{eV}^{-1} \mathrm{~cm}^{-3}$ for the back channel (24). Given the rather similar $\mathrm{N}_{\text {ot }}$ values for both oxides, the difference can be mainly ascribed to the coupling factor $\alpha$. Assuming that $S_{I}^{\text {acc }}=t^{2}{ }_{\text {ox }, 1} / t^{2}{ }_{\text {ox }, 2} S_{I, 2}$ and that $S_{I, 2}$ corresponds to the measured back-gate spectral density at $\mathrm{V}_{\mathrm{GS}}=0 \mathrm{~V}$, a coupling factor of 0.2 is derived, which is close to the theoretical value of 0.221 in this case.

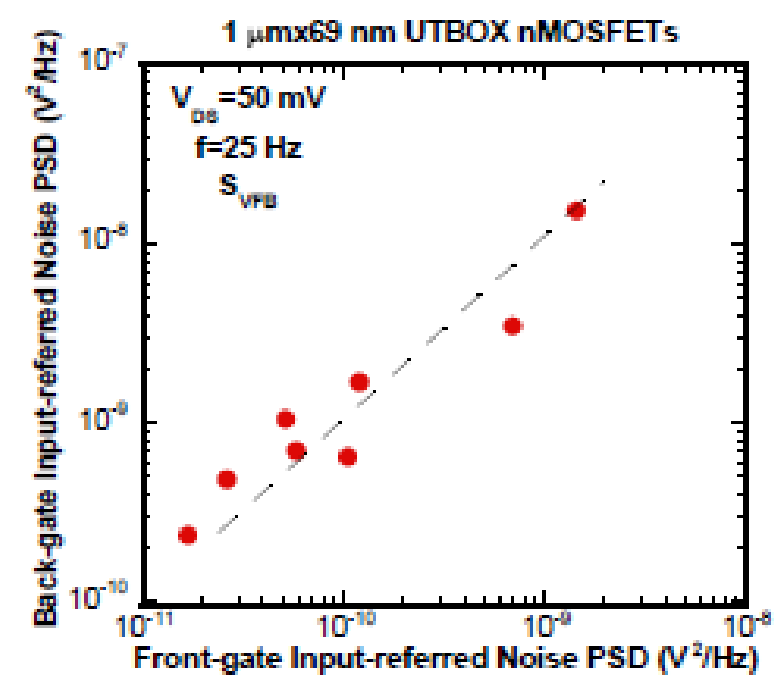

Fig. 4. Back- versus front-channel average input-referred noise PSD at flatband $\left(\mathrm{V}_{\mathrm{GS}} \sim \mathrm{V}_{\mathrm{T}}\right), \mathrm{f}=25 \mathrm{~Hz}$ and $\mathrm{V}_{\mathrm{DS}}=50 \mathrm{mV}$ for a set of $1 \mu \mathrm{m} \times 69 \mathrm{~nm}$ UTBOX nMOSFETs with $\mathrm{t}_{\mathrm{Si}} \approx 14 \mathrm{~nm}$ and $\mathrm{t}_{\mathrm{SiO} 2}=5 \mathrm{~nm}$. 
In case of the $6 \mathrm{~nm}$ film transistors, it is difficult to bias the back gate into accumulation. In that case, the coupling effect has to be accounted for both in the front and the back-channel noise $(24,28,29)$.

\section{GR noise and noise variability}

As shown in Fig. 1, excess GR noise, giving rise to a gate-voltage dependent Lorentzian spectrum can be found at higher frequencies. In some devices, on the other hand, GR noise also dominates at low frequencies, overwhelming the 1/f noise (Fig. 5). The high magnitude of this GR noise gives rise to a strong device-to-device variation in the LF noise PSD, as shown in Fig. 6: the normalized current noise PSD of similar UTBOX SOI nMOSFETs at a frequency $\mathrm{f}=25 \mathrm{~Hz}$ can vary over more than two decades at low drain currents $I_{D}$. Two groups of devices can be distinguished in Fig. 6: one with the lower PSD and corresponding with a $1 / \mathrm{f}^{\gamma}$ spectrum at low frequencies in Fig. $1(\gamma \sim 1)$ and a second set of devices, exhibiting a pronounced Lorentzian GR noise at low $\mathrm{f}$ (Fig. 5). Comparing Figs 1 and 5, it is clear that the excess GR noise is responsible for the one to two decades higher PSD observed in Fig. 6.

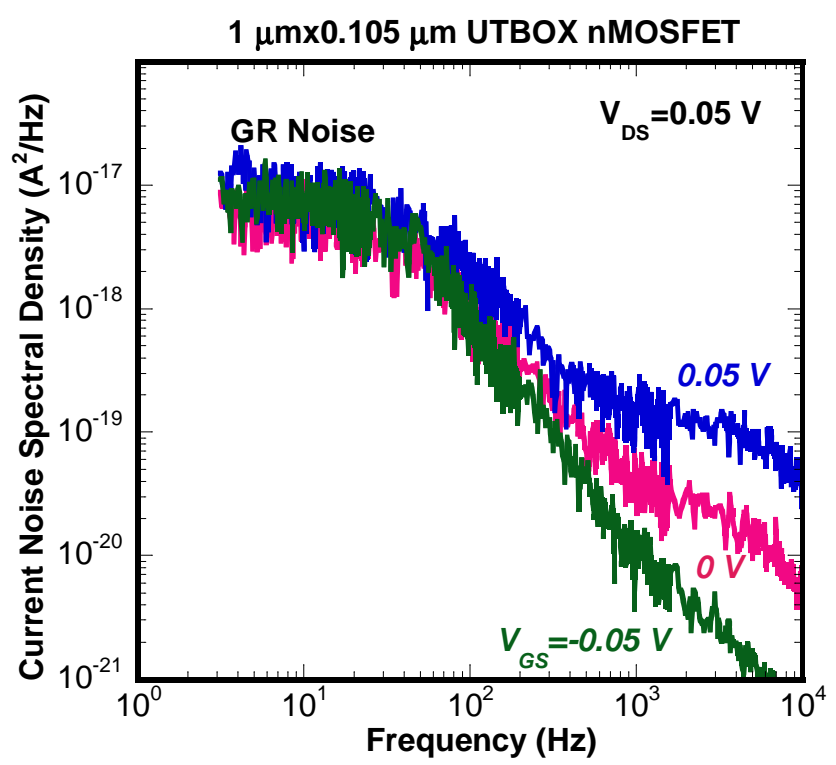

Fig. 5. Low-frequency noise spectra around $\mathrm{V}_{\mathrm{T}}$ for a $1 \mu \mathrm{mx} 0.105 \mu \mathrm{m}$ UTBOX SOI nMOSFET, exhibiting generation-recombination noise around $10 \mathrm{~Hz}$.

First, the origin of the excess GR noise in Fig. 5 has to be established. It is clear that the main parameters of the Lorentzian around $10 \mathrm{~Hz}$ in Fig. 5, namely, the plateau amplitude $S_{\mathrm{I}}(0)$ and the corner frequency $\left(\mathrm{f}_{0}\right)$ are fairly independent on the front gate voltage $\mathrm{V}_{\mathrm{GS}}$. This has been used in the past as an argument to ascribe the underlying trap centers as residing in the silicon depletion region in partially depleted or bulk MOSFETs, when they are operated in strong inversion $(8,9,30)$. Another argument in favor of this assignment is the fact that very similar Lorentzian noise is observed in the front and back-channel operation of the thin-film UTBOX nMOSFET (24). Since the same Lorentzian is observed in the front- and back-channel current noise, its origin should be common to both, i.e., the fully depleted silicon film. If it would correspond to a gate 
oxide trap, than the GR noise should only be present in the spectrum of the front or the back channel.

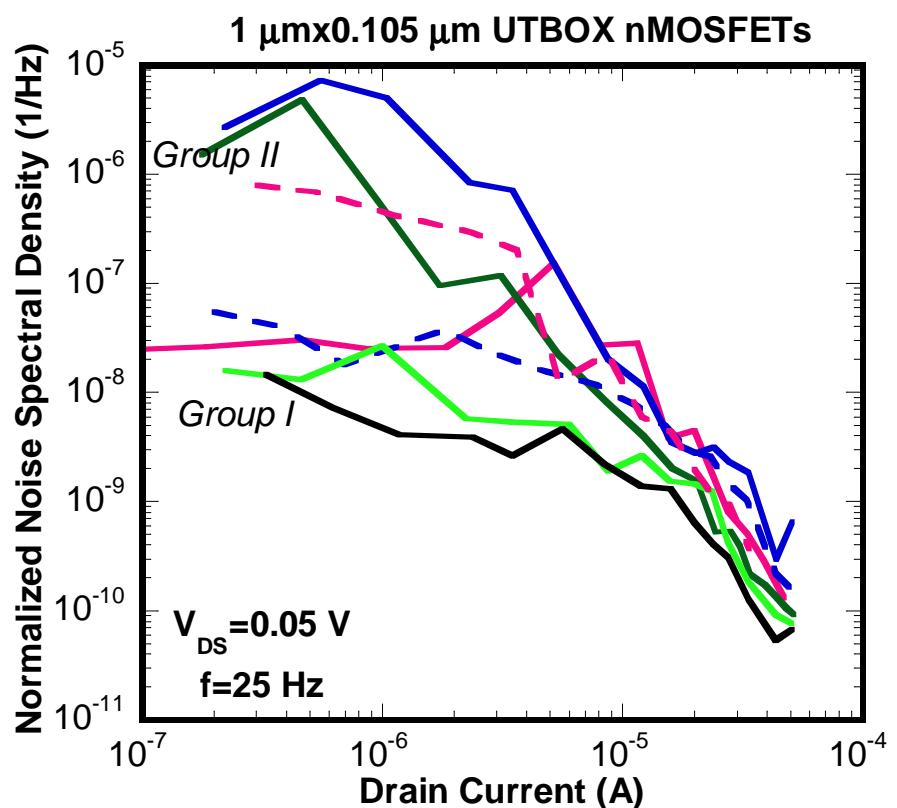

Fig. 6. Normalized noise current spectral density versus drain current at $\mathrm{f}=25 \mathrm{~Hz}$ and in linear operation for a set of FD SOI nMOSFETs, aligned across the vertical diameter in the center of an UTBOX wafer.

More recently, this interpretation of the gate-bias dependence of Lorentzian noise in has been challenged for FD SOI nMOSFETs $(31,32)$. Based on an extension of the classical model for GR noise in the depletion region of a MOSFET $(30,33)$, it has been shown that the Lorenztian parameters for a deep level center, which may exist in FD SOI or narrow FinFET type of structures, can show a strong variation with gate bias for a trap in a fully depleted Si layer. The model for the GR noise considers a PSD described by (30-33):

$$
\begin{aligned}
& S_{I_{d}}(f)=\frac{\overline{\delta I_{d}^{2}}}{\Delta f}=\frac{4 q^{2} Z}{L^{2}} \int_{x=0}^{L}\left(\mu_{n, e f f} R F(x)\right)^{2} \\
& \quad \times \int_{y=0}^{y_{\max }} N_{T}\left(1-\frac{y}{y_{\max }}\right)^{2} \frac{f_{t}\left(1-f_{t}\right) \tau}{1+(2 \pi f \tau)^{2}} d x d y
\end{aligned}
$$

In Eq. [5], $f_{t}$ is the Fermi function, defining the electron occupation of the trap level $E_{T}$, with concentration $\mathrm{N}_{T}$ and electron capture cross section $\sigma_{\mathrm{n}} \mathrm{Z}$ and $\mathrm{L}$ are the transistor width and length, respectively, while the integral of the Lorentzian spectrum is carried out over the thickness of the silicon film, from the front interface at $y=0$ to the buried interface at $y_{\max }$, which corresponds to the film or fin thickness. The first integral runs over the length of the transistor in the $\mathrm{x}$ direction and includes non-uniformities of the electric field $\mathrm{F}(\mathrm{x})$ and the effective electron mobility $\mu_{\mathrm{n}, \text { eff. }}$ For a channel with uniform doping profile and in linear operation, $\mathrm{F}(\mathrm{x})$ becomes $\mathrm{V}_{\mathrm{DS}} / \mathrm{L}$. Finally, the coupling factor $\mathrm{R}$ equals $\mathrm{C}_{\mathrm{N}} /\left(\mathrm{C}_{\mathrm{N}}+\mathrm{C}_{\mathrm{ox}}+\mathrm{C}_{\mathrm{Si}}\right)$, with $\mathrm{C}_{\mathrm{N}}, \mathrm{C}_{\mathrm{ox}}$ and $\mathrm{C}_{\mathrm{Si}}$ the capacitance densities of the inversion channel, the front-oxide and the depletion region ( $\mathrm{Si}$ film) and is included to avoid divergence of the PSD of Eq. [5] (31). 
The GR time constant in Eq. [5] is given by the Shockley-Read-Hall expression:

$$
\tau(x, y)=\left[c_{n}\left(n(x, y)+n_{t}\right)+c_{p}\left(p(x, y)+p_{t}\right)\right]^{-1}
$$

with $\mathrm{n}(\mathrm{x}, \mathrm{y}), \mathrm{p}(\mathrm{x}, \mathrm{y})$ the position-dependent free electron and free hole concentration, respectively; $\mathrm{n}_{\mathrm{t}}\left(\mathrm{p}_{\mathrm{t}}\right)$ the electron (hole) concentration when the Fermi level coincides with the trap level and $c_{n}$ and $c_{p}$ are the capture rates for electrons and holes, equal to the product of the respective capture cross section and thermal carrier velocity. The free carrier concentration profiles have been obtained from well-calibrated 2-dimensional device simulations of the UTBOX SOI nMOSFET input characteristics (31). For normal operation conditions of the nMOSFETs, the second term related to hole capture and emission in Eq. [6] can be neglected at most gate voltage conditions.

As exemplified by Fig. 7, the Lorentzian parameters exhibit a strong variation with the front-gate bias $\mathrm{V}_{\mathrm{GS}}$. This is explained by the strong dependence of the free electron concentration $\mathrm{n}(\mathrm{x}, \mathrm{y})$ on the Fermi level (Eq. [6]), which is modulated by the gate voltage. In case of UTBOX devices (or for independent double-gate FinFETs), also the back-gate bias can be used as a parameter to change the Fermi level position with respect to the trap level. In turn, this will impact on the free carrier density $\mathrm{n}(\mathrm{x}, \mathrm{y})$ in Eq. [6], which defines the SRH time constant. One can observe in Fig. 7 that the corner frequency of the Lorentzian given by $f_{0}=1 / 2 \pi \tau$ drastically increases going from low $V_{G S}$ in weak inversion to high inversion. At the same time, the plateau amplitude also increases significantly until a maximum is reached when $\mathrm{E}_{\mathrm{F}}$ crosses the trap level $\mathrm{E}_{\mathrm{T}}$. In principle, this can be used to perform trap spectroscopy at room temperature, by using the gatemodulation of the Lorentzian spectrum (31).

The point of interest here is the strong variation of the amplitude of the PSD calculated from Eq. [5] with gate voltage, further illustrated by Fig. 8 for traps in a FD silicon film with different activation energy and electron capture cross section. In other words, according to Fig. 8a, the amplitude of the Lorentzian noise at a fixed $\mathrm{V}_{\mathrm{GS}}$ can change over several orders of magnitude depending on the trap position with respect to the Fermi level and set by the front and/or back-gate voltage. Conversely, by changing the gate voltage, the Lorentzian PSD can be maximized when $\mathrm{E}_{\mathrm{F}}$ crosses $\mathrm{E}_{\mathrm{T}}$, giving a maximum contribution to the noise spectrum. This immediately implies that depending on the activation energy of the trap level, a significantly different Lorentzian noise can be obtained. In other words, a much higher Lorentzian amplitude will be obtained when traps are present in the silicon which are closer to the conduction band $\left(\mathrm{E}_{\mathrm{T}}-\mathrm{E}_{\mathrm{i}}=0.42 \mathrm{eV}\right)$ than when they are close to midgap $\left(\mathrm{E}_{\mathrm{T}}-\mathrm{E}_{\mathrm{i}}=0.12 \mathrm{eV}\right)$ in Fig. 8b. This corresponds with a significant source of variability in the low-frequency noise amplitude which differs from the one related with the standard RTN mechanism. It is typical for device structures with a FD silicon region between two gates, like in FD SOI or FinFETs on bulk or SOI substrates. Given the importance for $22 \mathrm{~nm}$ and below CMOS nodes of FinFETs, this is expected to become an important source of noise variability and should be accounted for in a similar way as the impact of RTN-induced dynamic variability. 


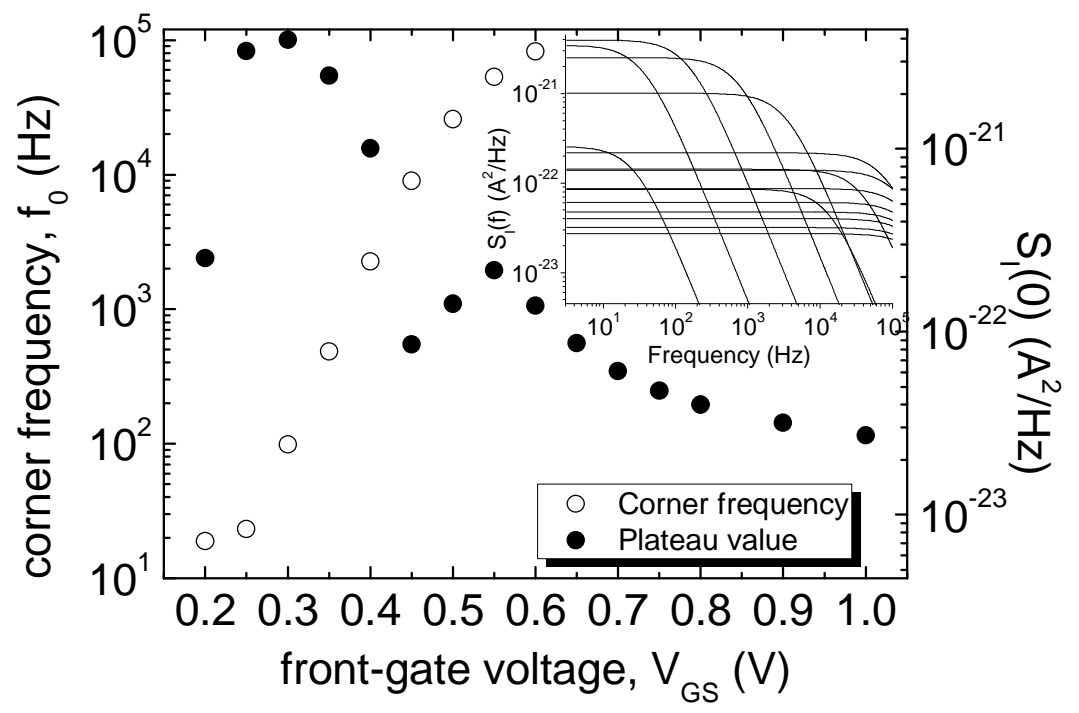

Fig. 7. Simulated corner-frequencies $f_{0}$ and plateau value $S_{I}(0)$ of the Lorentzian component as a function of the front-gate voltage. The back-gate voltage is $0 \mathrm{~V}$. Inset: detail of the variation of the corresponding Lorentzian spectra with gate voltage. $\mathrm{E}_{\mathrm{T}^{-}}$ $\mathrm{E}_{\mathrm{i}}=0.16 \mathrm{eV} ; \sigma_{\mathrm{n}}=2.5 \times 10^{-19} \mathrm{~cm}^{2}$ and $\mathrm{N}_{\mathrm{T}}=10^{17} \mathrm{~cm}^{-3}$.

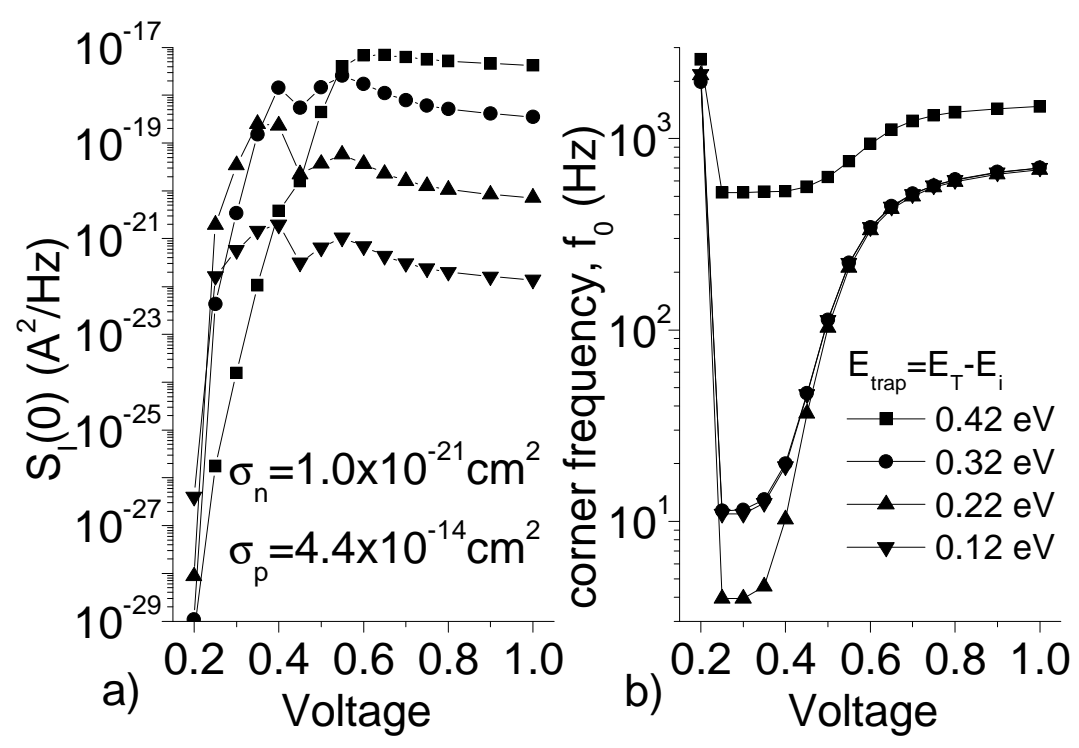

Fig. 8. Simulated plateau amplitude (a) and corner frequency (b) as a function of the front-gate voltage at zero back-gate, corresponding with different trap level positions with respect to the intrinsic Fermi level $\mathrm{E}_{\mathrm{i}}$. An electron capture cross section of $1.0 \times 10^{-21}$ $\mathrm{cm}^{2}$ and a hole cross section $\sigma_{\mathrm{p}}=4.4 \times 10^{-14} \mathrm{~cm}^{2}$ has been considered in the calculations. The existence of minority traps, such as the one analyzed in this figure can boost the noise variability. In this case, the peak seen at low voltages in (b) reflects the fact of a small number of electrons in the silicon and that the term related to hole capture and emission cannot be neglected 


\section{Random Telegraph Noise}

From the extracted trap concentrations, it is clear that the GR noise is generated by only a handful of traps - similar as the retention time, with similar activation energy (7). It is well-known that if only a few traps are present in the gate oxide, the $1 / \mathrm{f}$ noise transforms into so-called Random Telegraph Noise (RTN), also giving rise to a Lorentzian spectrum. The question arises: how to distinguish RTN from GR noise in the silicon film? It has recently been proposed that studying the noise in both the front and back-channel may enable to distinguish the different cases (24): when a similar Lorentzian is present in both spectra, like in Fig. 9, one can assume that the traps are present in the silicon film. If on the contrary, the Lorentzian is only found in the front or back-gate spectrum with the other channel accumulated, than one can conclude that the trap resides in the front or back-gate oxide. An example of RTN in the front-gate oxide is illustrated by Fig. 10: while the front-channel noise spectra exhibit a dominant Lorentzian at low frequencies, the spectra are more 1/f-like for the back-channel. In each case, the opposite channel was biased in accumulation for this $\mathrm{t}_{\mathrm{Si}}=14 \mathrm{~nm} \mathrm{nMOSFET}$, so that the effect of the corresponding traps on the noise was screened. In this case, it is concluded that RTN in the front oxide gives rise to this Lorentzian component. Notice also the much higher amplitude of the noise PSD, confirming the role of RTN in enhancing the noise variability.

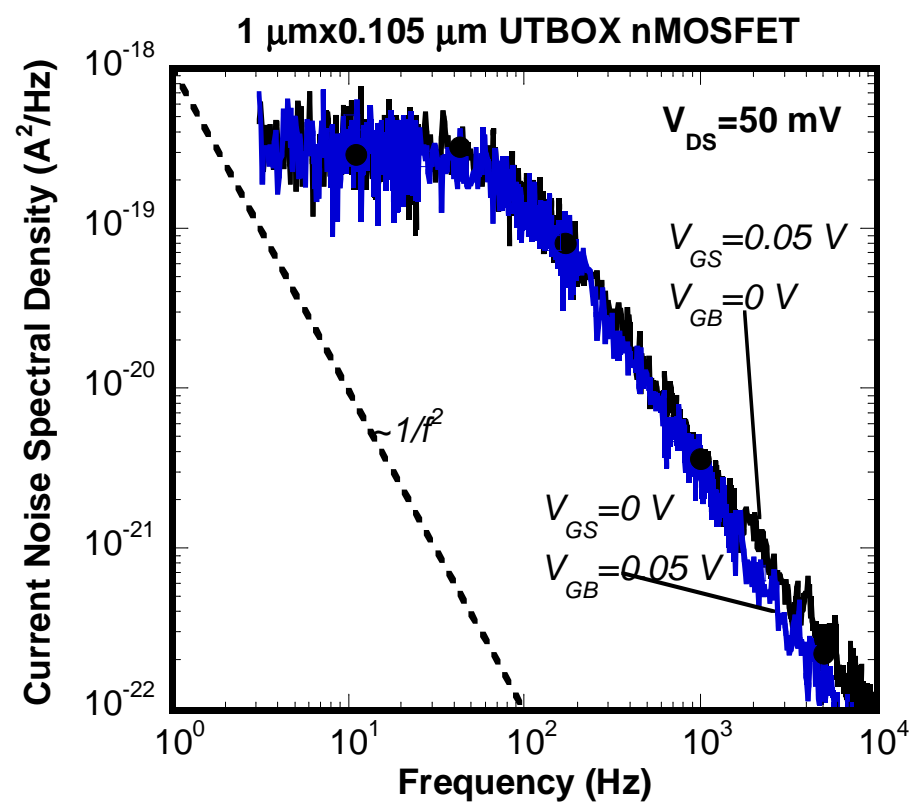

Fig. 9. Low-frequency noise spectra for a UTBOX SOI nMOSFET, operated at the frontgate with the back-gate $\mathrm{V}_{\mathrm{GB}}$ at $0 \mathrm{~V}$ or at the back-gate with the front-gate at $0 \mathrm{~V}$.

In order to obtain and idea of the activation energy of the responsible trap, the Lorentzian noise can for example be studied as a function of the temperature. This yields an activation energy $E_{a}=0.3 \mathrm{eV}$ for the gate oxide trap in Fig. 10 (34). Similar studies on a silicon film traps results in a shallower $E_{a}$ of $0.09 \mathrm{eV}$. Recent studies at cryogenic temperatures reveals the presence of several deep levels in the silicon film, which have a processing-induced character, i.e., some can be associated with implantation-induced defects (28). At the same time, the activation energy derived from temperature-dependent 
measurements is in good agreement with the data derived from the GR-noise spectroscopy method proposed in the previous section (31). At the same time, the thermal activation of the retention time gives similar values, in the range of 0.3 to $0.5 \mathrm{eV}$, depending on the processing details (7).

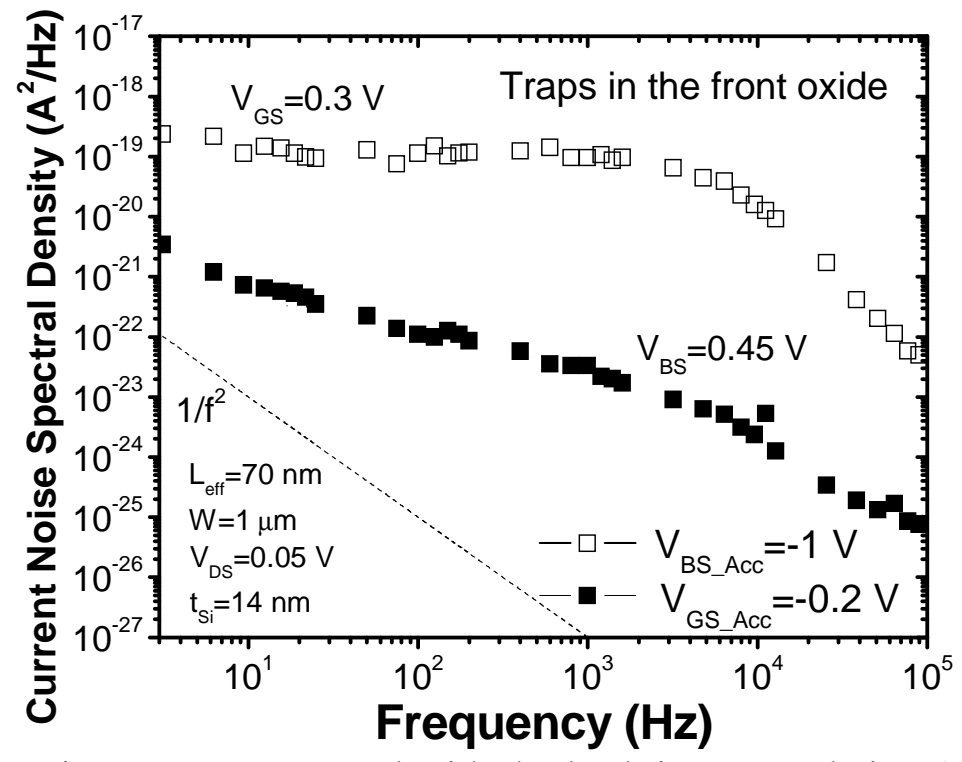

Fig. 10. Current noise spectra measured with the back in accumulation $\left(\mathrm{V}_{\mathrm{GS}, \text { acc }}\right)$ or with the front in accumulation $\left(\mathrm{V}_{\mathrm{BS}, \text { acc }}\right.$ ) for a $\mathrm{L}=69 \mathrm{~nm}$ and $\mathrm{t}_{\mathrm{Si}}=14 \mathrm{~nm}$ FD SOI nMOSFET.

\section{Conclusions}

In conclusion, it has been demonstrated that the LF noise PSD of thin film FD UTBOX SOI nMOSFETs exhibits both flicker noise and Lorentzian noise components, both in the front and the back channel. The flicker noise can be generally ascribed to trapping in the gate dielectric, whereby the magnitude depends on the gate oxide quality and type $\left(\mathrm{SiO}_{2}\right.$, high-k). The origin of the Lorentzian noise can be ascribed either to individual traps in the front or back gate dielectric or to defects residing in the fully depleted silicon film. This Lorentzian noise is the main origin of the wide sample-to-sample dispersion in the noise PSD of the studied devices. This may generally be extrapolated to other types of thin film or narrow fin fully depleted architectures like SOI or bulk FinFETs. This Lorentzian noise can be exploited to study the parameters of the deep levels in silicon or the gate oxide either by analyzing the gate-voltage or the temperature dependence. At the same time, this reveals useful information for the understanding of the retention time in UTBOX 1T FBRAM devices.

\section{Acknowledgements}

The imec Core Partners are gratefully acknowledged for financial support within the frame of the Advanced Devices program. Sara D. dos Santos and João Martino would like to thank Capes, Fapesp and CNPq for the financial support to develop this work. 


\section{References}

1. A. Asenov, R. Balasubramanian, A.R. Brown, and J.H. Davies, Superlatt. and Microstruct., 27, 411 (2000).

2. A. Asenov, R. Balasubramanian, A.R. Brown, and J.H. Davies, IEEE Trans. Electron Devices, 50, 839 (2003).

3. A. Ghetti, C. Monzio Compagnoni, A.S. Spinelli, and A. Visconti, IEEE Trans. Electron Devices, 56, 1746 (2009).

4. T. Mizumo, J. Okamura, and A. Toriumi, IEEE Trans. Electron Devices, 41, 2216 (1994).

5. C. Lee, A.T. Putra, K. Shimizu, and T. Hiramoto, Jpn. J. Appl. Phys., 49, 04DC01-1 (2010).

6. T. Nicoletti, M. Aoulaiche, L.M. Almeida, S.D. dos Santos, J.A. Martino, A. Veloso, M. Jurczak, E. Simoen, and C. Claeys, IEEE Electron Device Lett., 33, 940 (2012).

7. M. Aoulaiche, E. Simoen, M. Veloso, P. Blomme, G. Groeseneken, M. Jurczak, T. Nicoletti, and L. Mendes Almeida, IEEE Trans. Electron Devices, 59, 2167 (2012).

8. I. Lartigau, J.M. Routoure, W. Guo, B. Cretu, R. Carin, A. Mercha, C. Claeys and E. Simoen, J. Appl. Phys., 101, 104511-1 (2007).

9. R. Talmat, H. Achour, B. Cretu, J-M. Routoure, A.Benfdila, R. Carin, N. Collaert, A. Mercha, E. Simoen and C. Claeys, Solid-State Electron., 70, 20 (2012).

10. M.J. Kirton and M.J. Uren, Adv. in Phys. 38, 367 (1989).

11. G. Ghibaudo, O. Roux, and J. Brini, Phys. Stat. Sol. A, 132, 501 (1992).

12. D. Lopez, S. Haendler, C. Leyris, G. Bidal, and G. Ghibaudo, IEEE Trans. Electron Devices, 58, 2310 (2011).

13. E.G. Ionannidis, S. Haendler, A. Bajolet, T. Pahron, N. Planes, F. Arnaud, R.A. Bianchi, M. Haond, D. Golanski, J. Rosa, C. Fenouillet-Beranger, P. Perreau, C.A. Dimitriadis, and G. Ghibaudo, In: Tech. Dig. IEDM 2011 (The IEEE, New York), p. 449 (2011).

14. M. Agostinelli, J. Hicks, J. Xu, B. Woolery, K. Mistry, K. Zhang, S. Jacobs, J. Jopling, W. Yang, B. Lee, T. Raz, M. Mehalel, P. Kolar, Y. Wang, J. Sandford, D. Pivin, C. Peterson, M. DiBattista, S. Pae, M. Jones, S. Johnson, and G. Subramanian, In: Tech. Dig. IEDM 2005, (The IEEE, New York), p. 655 (2005).

15. H. Kurata, K. Otsuga, A. Kotabe, S. Kajiyama, T. Osabe, Y. Sasago, S. Narumi, K. Tokami, S. Kamohara, and O. Tsuchiya, IEEE J. Solid-St. Circ., 42, 1362 (2007).

16. A. Ohata, A. Toriumi, M. Iwase, and K. Natori, J. Appl. Phys., 68, 200 (1990).

17. E. Simoen, B. Dierickx, C.L. Claeys and G.J. Declerck, IEEE Trans. Electron Devices, 39, 422 (1992).

18. H.H. Mueller, U. Schirl, and M. Schulz, Microelectron. Eng., 28, 163 (1995).

19. H.H. Mueller and M. Schulz, J. Appl. Phys., 79, 4178 (1996).

20. P. Zhang, Y.Q. Zhuang, Z.F. Ma, L. Bao, L. Du, and J.L. Bao, Semicond. Sci. Technol., 23, 125037-1 (2008).

21. M.-J. Chen, C.-C. Lee, and M.-P. Lu, J. Appl. Phys., 103, 034511-1 (2008).

22. E. Simoen, A. Mercha, C. Claeys, N. Lukyanchikova, and N. Garbar, IEEE Trans. Electron Devices, 51, 1008 (2004). 
23. S.D. dos Santos, E. Simoen, V. Strobel, B. Cretu, J.-M. Routoure, R. Carin, M. Aoulaiche, M. Jurczak, J.A. Martino, and C. Claeys, In: the Proc. Of ICSICT, Xi'an, China, 30 Oct-2 Nov. 2012.

24. S.D. dos Santos, T. Nicoletti, J.A. Martino, M. Aoulaiche, A. Veloso, M. Jurczak, E. Simoen, and C. Claeys, IEEE Trans. Electron Devices, 60, 444 (2013).

25. G. Ghibaudo, O. Roux, C. Nguyen-Duc, F. Balestra, and J. Brini, Phys. Stat. Sol. A 124, 571 (1991).

26. T. Boutchacha, G. Ghibaudo, G. Guégan, and M. Haond, J. Non-Cryst. Solids 216, 123 (1997).

27. G. Ghibaudo, L. Zafari, and J. Jomaah, Solid-St. Electron., 51, 292 (2007).

28. V. Strobel, B. Cretu, S.D. dos Santos, E. Simoen, J.-M. Routoure, R. Carin, M. Aoulaiche, M. Jurczak, J.A. Martino, and C. Claeys, In: Abstr. EUROSOI 2013, Paris (France), 21-23 January 2013.

29. S.D. dos Santos, J.A. Martino, V. Strobel, B. Cretu, J.-M. Routoure, R. Carin, E. Simoen, M. Aoulaiche, M. Jurczak, and C. Claeys, to be published in the Proc. Of CSTIC 2013, Shanghai (China), March 2013.

30. N. Lukyanchikova, in Noise and Fluctuations Control in Electronic Devices, edited by A. Balandin American Scientific, Riverside, CA, 2002.

31. A. Luque Rodríguez, J.A. Jiménez Tejada, S. Rodriguez-Bolívar, M. Aoulaiche, C. Claeys and E. Simoen, IEEE Trans. Electron Devices, 59, 2780 (2012).

32. E. Simoen, R. Tomasso, L. Pantisano, A. Luque Rodriguez, J.A. Jiménez Tejada, M. Aoulaiche, A. Veloso, M. Jurczak, R. Krom, J. Mitard, Ch. Caillat, P. Fazan, F. Crupi, and C. Claeys, in: Tech. Dig. IEDM 2012 (The IEEE, New York), p. 28.7 (2012).

33. L.D. Yau and C. T. Sah, IEEE Trans. Electron Devices 16, 170 (1969).

34. S.D. dos Santos, J. A. Martino, M. Aoulaiche, M. Jurczak, E. Simoen, and C. Claeys, abstract submitted for presentation at ICNF 2013, Montpellier (France), 24-28 June, 2013. 